Copyright by the Acoustical Society of America. Pan, J., Snyder, S. D., Hansen, C. H., \& Fuller, C. R. (1992). Active control of far-field sound radiated by a rectangular panel - A general analysis. Journal of the Acoustical Society of America, 91(4), 2056-2066. doi: 10.1121/1.403691

\title{
Active control of far-field sound radiated by a rectangular panel-A general analysis
}

\author{
Jie Pan, ScottD. Snyder, and Colin H. Hansen \\ Department of Mechanical Engineering, University of Adelaide, GPO Box 498, South Australia, \\ 5001, Australia \\ Christopher R. Fuller \\ Department of Mechanical Engineering, Virginia Polytechnic Institute and State University, Blacksburg, \\ Virginia 24061
}

(Received 5 November 1990; accepted for publication 18 November 1991)

\begin{abstract}
In this paper a general analysis is presented for the active control of the far-field harmonic sound radiated by a rectangular panel that is built into an infinite baffle. In this analysis, the panel vibration may be generated by either airborne sound (incident sound field) or by structure borne vibrations. The far-field radiated sound is controlled either by acoustical sources or vibration sources. Minimization of both the local sound pressure and the total power output is considered. Analytical results for the particular case involving minimization of the sound pressure at a single point are compared with experimental data. The physical mechanisms involved for different control sources (vibration or acoustic) are demonstrated analytically. For the case of vibration control sources, the panel modal velocity components are adjusted to produce far-field sound control. This can be done either by decreasing their amplitudes, and/or by changing the temporal phases of the panel modes. However, for acoustic control sources, the far-field sound is minimized by alteration of the radiation impedance seen by the vibrating panel and the control sources.
\end{abstract}

PACS numbers: 43.50.Ki, 43.50.Gf, 43.40.Vn

\section{INTRODUCTION}

While the use of active noise control in ducts has met with considerable success, ${ }^{1}$ its application to three-dimensional sound fields (as opposed to one-dimensional plane waves) has not. Recent work ${ }^{2}$ has demonstrated that the acoustic control mechanism in a duct is the change in the radiation impedance of the noise source, caused by the introduction of a secondary disturbance, thereby decreasing the acoustic power flow under controlled conditions. Other analytical work ${ }^{3}$ has demonstrated these concepts for monopole sources radiating into free space.

Many of the low-frequency noise problems targeted as possible recipients of active control involve the radiation of sound from vibrating surfaces. This can either be radiation into free space, as with a transformer, ${ }^{4}$ or transmission into a coupled structural/acoustic system, as with an aircraft fuselage ${ }^{5}$ or automobile interior. ${ }^{6}$ The main body of work has centered around the use of acoustic sources for control. It has been found that the use of a single acoustic source produces a small "quiet zone," at the expense of increased sound levels elsewhere. ${ }^{7}$ Surrounding the vibrating surface with acoustic sources can, however, result in global attenuation of the sound levels. ${ }^{4}$

Applying the understanding of the mechanism of active noise control from the one-dimensional case of a duct to three-dimensional systems, it can be surmised that for global sound attenuation to be achieved, the total sound power radiated by the noise source must be reduced. If acoustic sources are to be successful in controlling sound radiation from a vibrating surface, they must be able to significantly alter the radiation impedance "seen" by the source. Alternatively, vibration actuators such as electrodynamic shakers ${ }^{5}$ or piezoelectric ceramics ${ }^{8,9}$ may also be used successfully as control sources on a vibrating surface if they can decrease the panel surface velocity and/or alter the relative temporal phases of the structural modes, and hence change the overall phase between the surface velocity and pressure. This has been demonstrated experimentally ${ }^{10}$ for sound radiation into free space, and theoretically ${ }^{11}$ for sound transmission into a rectangular enclosure.

Previous theoretical investigations of the active control of sound radiation from a panel into free space have considered the use of either acoustic control sources ${ }^{12}$ or vibration (point force) control sources. ${ }^{13}$ The following is a general analysis of the use of control forces or acoustic sources to control harmonic sound radiation from a simply supported baffled panel. The purpose of the analysis is to be able to predict the maximum radiated sound power reduction, which would be achievable using an ideal controller and a particular control source arrangement. Experimental data are provided to verify the theory. Although the following analysis applies to a panel excited by an incident acoustic field or by point forces, for the sake of brevity, experimental results are only provided for point force excitation.

\section{PANEL RESPONSE}

Figure 1 shows the coordinates of a simply supported rectangular panel in an infinite baffle. A vibration field on 


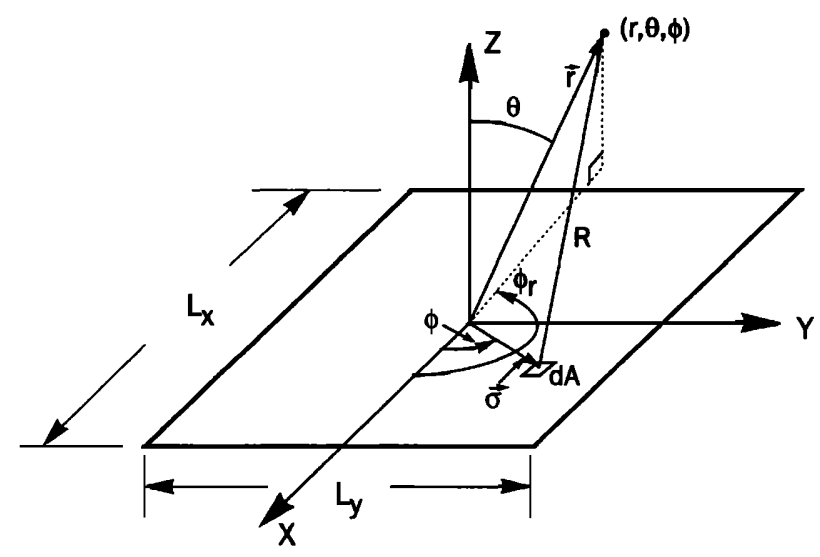

FIG. 1. Coordinate system of a simply supported panel in a baffle.

the panel can be generated either by an incident sound field or by a vibration force acting on the panel surface. This distributed panel velocity results in a sound field radiated into the upper semi-infinite space.

If the panel is thin and isotropic, its displacement response to a total distributed sound pressure $p_{\text {total }}$ on both panel surfaces can be described by ${ }^{14}$

$$
\rho h \frac{\partial^{2} W}{\partial t^{2}}+\frac{E h^{3}}{12\left(1-\mu^{2}\right)} \nabla^{4} W=p_{\text {total }},
$$

where $\rho, h, E$, and $\mu$ are the density, thickness, Young's modulus, and Poisson's ratio of the panel, respectively. Here $p_{\text {total }}$ is made up of primary and control pressures that result from either sound fields or point forces.

The panel velocity amplitude $\bar{v}(\sigma)$ at location $\sigma$ can be expressed by normal mode expansion. For the simply supported rectangular panel,

$$
\bar{v}(\boldsymbol{\sigma}) \exp (j \omega t)=\frac{\partial W}{\partial t}=\sum_{m, n} \bar{v}_{m, n} S_{m, n}(\boldsymbol{\sigma}) \exp (j \omega t),
$$

where $m$ is the mode order in the $X$ direction and $n$ is the mode order in the $Y$ direction. Here, $\bar{v}_{m, n}$ is the modal velocity component of $(m, n)$ mode. With the origin of the coordinate system in the center of the panel, the normal mode shape function $S_{m, n}(\sigma)$ is

$$
S_{m, n}(\sigma)=\sin \left(\frac{m \pi x}{L_{x}}+\frac{m \pi}{2}\right) \sin \left(\frac{n \pi y}{L_{y}}+\frac{n \pi}{2}\right) .
$$

Substituting Eq. (2) into Eq. (1), introducing the panel modal damping, and using the orthogonality property of the functions $S_{m, n}(\sigma)$, the panel modal velocity component matrix $\left[\bar{v}_{m, n}\right](M \times 1)$ can be expressed by

$$
\left[\bar{v}_{m, n}\right]=\left[\boldsymbol{Y}^{P}\right]\left[\boldsymbol{P}_{m, n}^{t}\right],
$$

where $M$ is the total number of panel modes considered. The panel modal input admittance matrix $\left[Y^{P}\right]$ is an $M \times M$ diagonal matrix with its diagonal element as

$$
\boldsymbol{Y}_{m, n}^{P}=\frac{j \omega A_{f}}{\boldsymbol{M}_{m, n}^{P}\left(\omega_{m, n}^{2}+j \eta_{m, n} \omega_{m, n}^{2}-\omega^{2}\right)},
$$

where $M_{m, n}^{P}=\rho h A_{f} \Lambda_{m, n}$ is the panel modal mass and $A_{f}$ is the panel surface area. The normalizing factor $\Lambda_{m, n}$ is defined as

$$
\Lambda_{m, n}=\frac{1}{A_{f}} \int_{A_{f}} S_{m, n}(\boldsymbol{\sigma}) S_{m, n}(\boldsymbol{\sigma}) d \sigma .
$$

Here, $\eta_{m, n}$ is the loss factor of the $(m, n)$ panel mode and it is related to the $60-\mathrm{dB}$ modal decay time $T_{m, n}$ by $\eta_{m, n}=4.4 \pi / T_{m, n} \omega_{m, n}$.

Here $\left[P_{m, n}^{t}\right]$ is an $(M \times 1)$ matrix and its $i$ th element is the product of the total pressure $p_{\text {total }}$ and the shape function of the $i$ th panel mode at the point of application of the force, integrated over the panel surface. Here $\left[P_{m, n}^{t}\right]$ is represented as follows:

$$
\left[P_{m, n}^{t}\right]=\left[P_{1}^{t} \cdots P_{M}^{t}\right]^{T},
$$

where the $i$ th element [corresponding to the $(m, n)$ mode] is

$$
P_{i}^{t}=\frac{1}{A_{f}} \int_{A_{f}} S_{m, n}(\boldsymbol{\sigma}) p_{\text {total }}(\boldsymbol{\sigma}) d \boldsymbol{\sigma} .
$$

\section{SOUND PRESSURE ON THE PANEL SURFACES}

The total sound pressure $p_{\text {total }}$ distributed on both panel surfaces can be expressed as

$$
p_{\text {total }}=p_{\mathrm{pri}}^{0^{-}}+p_{\mathrm{con}}^{0^{-}}+p_{\mathrm{rad}},
$$

where $p_{\mathrm{pri}}^{0^{-}}$is the pressure due to the primary force, $p_{\mathrm{con}}^{0^{-}}$is the pressure due to the control forces, and $p_{\text {rad }}$ is the radiation loading pressure. The sound pressure $p_{\text {rad }}$ is the distributed sound pressure evaluated on the panel surfaces due to all radiation loading terms. These loading terms are the sound pressure loading on the panel surface by the acoustic control sources and the loading due to panel radiation into the positive and negative $Z$ spaces. In this model, all the loading terms are assumed to be a second-order effect on the panel velocity distribution, and are ignored; thus $p_{\text {rad }}=0$.

For a plane wave incident from any arbitrary direction, the sound pressure distribution on the back surface of the panel $p_{\mathrm{pri}}^{0^{-}}$can be approximated by the superposition of the incident and reflected sound pressure:

$$
p_{\text {pri }}^{0^{-}}=2 p_{\text {pri }} \text {. }
$$

The incident sound pressure can be written as

$$
p_{\text {pri }}=p_{\text {ino }} \exp (j \omega t-j \mathbf{k} \cdot \boldsymbol{\sigma}),
$$

where $\mathbf{k}$ is a wave number vector of the incident wave and $\sigma$ is the location vector of the observation point on the panel surface. Here, $p_{\text {ino }}$ is a complex amplitude.

For a primary vibration force input only, such as a mechanical shaker or electromagnetic driver at $\sigma_{i_{0}}$, the effective pressure induced on the panel at location $\sigma$ can be written as

$$
p_{\text {pri }}(\boldsymbol{\sigma})=f_{i_{0}}^{\text {in }} \delta\left(\boldsymbol{\sigma}-\sigma_{i_{0}}\right) \exp (j \omega t),
$$

where $f_{i_{0}}^{\text {in }}=f_{0_{i_{0}}}^{\text {in }} \exp \left(j \phi_{i_{0}}^{\text {in }}\right)$ is the complex force at position $\sigma_{i_{0}}$ on the panel surface and $\delta\left(\sigma-\sigma_{i_{0}}\right)$ is a Dirac delta function.

If the primary input forces are discrete point forces, which can be produced by $M_{1}$ drivers, the quantity $p_{\mathrm{pri}}^{0^{-}}$due to these forces is the superposition of all the resultant pressures; that is,

$$
p_{\text {pri }}^{0^{-}}=\sum_{i_{0}=1}^{M_{1}} f_{i_{0}}^{\text {in }} \delta\left(\sigma-\sigma_{i_{0}}\right) \exp (j \omega t) .
$$


For the control point force contributions, the pressure $p_{\text {con }}^{0^{-}}$generated at the panel back surface has a similar form to that for the input primary vibration drivers. If $M_{2}$ control actuators located at $\sigma_{i_{1}}\left(i_{1}=1, \ldots, M_{2}\right)$ are used, the pressure distribution on the panel surface is

$$
p_{\mathrm{con}}^{0^{-}}=\sum_{i_{1}=1}^{M_{2}} f_{i_{1}}^{\mathrm{con}} \delta\left(\sigma-\sigma_{i_{1}}\right) \exp (j \omega t) .
$$

\section{FAR-FIELD SOUND PRESSURE}

\section{A. Vibration control sources only}

With vibration control sources only, the far-field sound pressure is generated entirely by the vibration response of the panel, and may be described using the Rayleigh integral. That is, the radiated sound pressure at angular frequency $\omega$ and location $r$ is given by ${ }^{14}$

$p_{v}(\mathbf{r}, \omega)=\frac{j k \rho_{0} c_{0}}{2 \pi} \int_{A_{f}} \frac{\bar{v}(\boldsymbol{\sigma}) \exp [j(\omega t-k R)]}{R} d A$,

where $k$ is the wave number of the radiated acoustic field, $\omega$ is the angular frequency of the excitation, $\rho_{0}$ is the density of air, $c_{0}$ is the speed of sound in free space, $\bar{v}(\sigma)$ is the velocity amplitude at point $\sigma$, and $R$ is the distance from the element $d A$ to the observation point (see Fig. 1). For the far-field condition ( $r \gg \max \left[L_{x}, L_{y}\right]$ ), $R$ in the denominator (amplitude part of the radiated pressure) can be approximated by $R \approx|\mathbf{r}|$, where $|\mathbf{r}|$ is the distance from the origin to the observation point. The $R$ in the phase part can be approximated as $^{14}$

$$
R \approx|\mathbf{r}|-|\boldsymbol{\sigma}| \cos (\boldsymbol{\sigma} \cdot \mathbf{r}),
$$

where $\cos (\boldsymbol{\sigma} \cdot \mathbf{r})$ is the cosine of the angle between vectors $\sigma$ and $\mathbf{r}$ and

$$
\begin{aligned}
\cos (\boldsymbol{\sigma} \cdot \mathbf{r})=(\boldsymbol{\sigma} \cdot \mathbf{r}) /|\boldsymbol{\sigma}||\mathbf{r}|= & \cos \phi \sin \theta \cos \phi_{r} \\
& +\sin \phi \sin \theta \sin \phi_{r} .
\end{aligned}
$$

Therefore Eq. (16) becomes

$$
R \approx|\mathbf{r}|-\frac{1}{k}\left[\left(\frac{\alpha x}{L_{x}}\right)+\left(\frac{\beta y}{L_{y}}\right)\right],
$$

where

$$
\begin{aligned}
& \alpha=k L_{x} \sin \theta \cos \phi_{r}, \\
& \beta=k L_{y} \sin \theta \sin \phi_{r},
\end{aligned}
$$

and $L_{x}, L_{y}$ are the panel dimensions. As shown in Fig. 1, $\theta$ and $\phi_{r}$ are the elevation and azimuth angles of vector $r$, while $\phi$ is the azimuthal angle of the vector $\sigma$ from the positive $X$ axis.

Using Eqs. (2) and (15), the sound pressure at the farfield observation point $\mathbf{r}$ due to the panel vibration response can be expressed in matrix form as

$$
p_{v}(\mathbf{r}, \omega)=\left[Z_{\mathrm{rad}}\right]^{T}\left[\bar{v}_{m, n}\right],
$$

where $\left[Z_{\mathrm{rad}}\right]$ is the modal radiation transfer function matrix $(M \times 1)$, and its $i$ th element, corresponding to the $i$ th mode [or the $(m, n)$ mode], gives the following for the relationship between the velocity distribution for that mode and the radiated sound pressure due to that mode,

$$
\begin{aligned}
Z_{i}^{\mathrm{rad}}= & \frac{j k \rho_{0} c_{0} \exp [j(\omega t-k|\mathbf{r}|)]}{2 \pi|\mathbf{r}|} \\
& \times \int_{A_{f}} S_{m, n}(\boldsymbol{\sigma}) \exp \left[j\left(\frac{\alpha x}{L_{x}}\right)+j\left(\frac{\beta y}{L_{y}}\right)\right] d A,
\end{aligned}
$$

where $S_{m, n}(\sigma)$ is the mode shape of the $(m, n)$ th mode evaluated at $\sigma$. After the transformation of $\left(x=x^{\prime}-L_{x} / 2 ; y=y^{\prime}-L_{y} / 2\right)$, Wallace's result ${ }^{15}$ can be used for this integration. Equation (22) can be rewritten as

$$
\begin{aligned}
Z_{i}^{\mathrm{rad}}= & \frac{j k \rho_{0} c_{0} \exp [j(\omega t-k|\mathbf{r}|)]}{2 \pi|\mathbf{r}|} \frac{A_{f}}{m n \pi^{2}}\left(\frac{(-1)^{m} e^{j \alpha}-1}{(\alpha / m \pi)^{2}-1}\right) \\
& \times\left(\frac{(-1)^{n} e^{j \beta}-1}{(\beta / n \pi)^{2}-1}\right) \exp \left[-j\left(\frac{\alpha}{2}\right)-j\left(\frac{\beta}{2}\right)\right] .
\end{aligned}
$$

In Eq. (21), the panel modal velocity matrix $\left[\bar{v}_{m, n}\right]$ and thus the total far-field sound pressure $p_{v}(r, \omega)$ includes both the contribution from the primary source and that from the control sources. Note that the modal radiation transfer function matrix $\left[Z_{\mathrm{rad}}\right]$ is independent of the effect of control sources. Thus minimization of the sound field by use of vibration control sources is achieved by modification of the panel modal velocities $\left[\bar{v}_{m, n}\right]$. This can be done in either one or both of two ways; the control forces can either minimize the modal amplitudes of all modes contributing significantly to the far-field sound pressure or they can adjust the amplitudes and the phases of each component in $\left[\bar{v}_{m, n}\right]$ so that the superimposed total sound field is minimized. Note that in this case, the overall rms panel velocity levels are not necessarily reduced.

\section{B. Acoustical control sources only}

If $N$ monopole sound sources are used as active control sources, the far-field sound radiated by each monopole can be determined by considering the Green's functions of the source and its mirror image

$$
\begin{aligned}
p_{a}(\mathbf{r}, \omega)_{i_{2}}= & \left(j k \rho_{0} c_{0} u_{i_{2}} / 4 \pi r_{i_{2}}^{+}\right) \exp \left[j\left(\omega t-k r_{i_{2}}^{+}\right)\right] \\
& +\left(j k \rho_{0} c_{0} u_{i_{2}} / 4 \pi r_{i_{2}}^{-}\right) \exp \left[j\left(\omega t-k r_{i_{2}}^{-}\right)\right],
\end{aligned}
$$

where $u_{i_{2}}$ is the volume velocity of the $i_{2}$ th monopole, and $r_{i_{2}}^{+}$and $r_{i_{2}}^{-}$are the distances from the source and its image to the observation point, respectively. If the monopole sources are close to the panel compared with the far-field observation point, then $r_{i_{2}}^{+}$and $r_{i_{2}}^{-}$in the denominator (the amplitude part) can be approximated by $r$.

The superposition of the sound pressures in the far field from the $N$ secondary acoustic sources can be written as

$$
p_{a}(\mathbf{r}, \omega)=\left[Z_{\text {mono }}\right]^{T}[u],
$$

where $\left[Z_{\text {mono }}\right]$ is the radiation transfer function matrix $(N \times 1)$, and its $i_{2}$ th element, corresponding to the $i_{2}$ th acoustic source for the far-field case, is

$$
\begin{aligned}
Z_{i_{2}}^{\text {mono }}= & \left(j k \rho_{0} c_{0} / 4 \pi r\right) \\
& \times\left[\exp \left(-j k r_{i_{2}}^{-}\right)+\exp \left(-j k r_{i_{2}}^{+}\right)\right] ;
\end{aligned}
$$

[ $u$ ] is the $(N \times 1)$ volume velocity matrix for the $N$ monopoles; its $i_{2}$ th element is the volume velocity of the $i_{2}$ th mon- 
opole. The far-field sound pressure is the sum of the pressure due to the vibration response of the panel and the pressure due to the acoustic control sources. It can be written as

$$
p(\mathbf{r}, \omega)=p_{a}(\mathbf{r}, \omega)+p_{v}(\mathbf{r}, \omega),
$$

where $p_{v}(\mathbf{r}, \omega)$ is defined by Eq. (21) with the contribution due to the vibration control sources excluded.

In this case, where only acoustic control sources are used, neither the panel velocity field $\left[\bar{v}_{m, n}\right]$ nor the volume velocities $[u]$ of the acoustic control sources can be significantly altered when the far-field sound pressure is minimized. This is because the sound induced forces on them are second order in magnitude compared with the internal driving forces of the acoustic and panel radiation sources. Therefore, the modal velocity adjustment control mechanism involved when vibration control sources are used cannot be the mechanism involved here.

The mechanism involved here may be the mutual adjustment of the sound pressure field in front of the primary and control sources. Optimal control can be achieved by adjusting the magnitude and phase of the sound pressure in front of each source, such that the product of the source velocity and the pressure (or sound intensity) at each source has minimum or negative real components. This effectively results in an alteration of the radiation transfer functions $\left[Z_{\mathrm{rad}}\right]$ and $\left[Z_{\text {mono }}\right]$. This mechanism can be demonstrated by placing a point acoustic control source close to a monopole acoustic source in a free field. It can be shown that the total radiated far-field sound pressure can be reduced by adjusting the control source so that it has the same volume velocity magnitude and opposite phase. The resultant sound field will be characteristic of a dipole source, which effectively has a smaller radiation impedance. The volume velocity can be adjusted further by using an optimization method, so that the control source becomes a sound absorber; ${ }^{16}$ in this case, the far-field sound pressure can be reduced even more.

\section{MINIMIZATION OF SOUND PRESSURE AT A POINT(S)}

The total sound pressure level at any point in the far field of the panel, $p(\mathbf{r}, \omega)$, is equal to the sum of the pressures of the primary noise source and the control source(s):

$$
p(\mathbf{r}, \omega)=p^{P}(\mathbf{r}, \omega)+p^{S}(\mathbf{r}, \omega) .
$$

Thus, the pressure amplitude squared is

$$
\begin{aligned}
|p(\mathbf{r}, \omega)|^{2}= & p^{S}(\mathbf{r}, \omega) p^{S}(\mathbf{r}, \omega)^{*}+p^{P}(\mathbf{r}, \omega) p^{S}(\mathbf{r}, \omega)^{*} \\
& +p^{S}(\mathbf{r}, \omega) p^{P}(\mathbf{r}, \omega)^{*}+p^{P}(\mathbf{r}, \omega) p^{P}(\mathbf{r}, \omega)^{*},
\end{aligned}
$$

where the asterisk denotes the complex conjugate.

It can be deduced from Eq. (29) that the total sound pressure squared at any point in the far field is a quadratic function of the pressure produced by the control source(s). Assuming that the acoustic control source(s) has an infinite internal impedance [constant volume velocity source(s)], or the vibration source(s) has a negligible internal impedance [constant force source(s) ], Eq. (29) can be reexpressed in terms of the complex control forces for vibration control sources, or in terms of the complex source volume velocities for acoustic control sources. The above assumptions are usually closely approximated by common acoustic sources and electromagnetic and piezoceramic vibration sources. The assumptions imply that for the case of vibration sources, the force output is unaffected by other forces acting on the panel. For acoustic control sources, the volume velocity of the source is unaffected by the presence of other sound fields.

Consider, first, the total pressure produced by the primary noise source operating alone. From Eqs. (4), (7), (8), and $(21)$, this is equal to

$$
p^{P}(\mathrm{r}, \omega)=\left[Z_{\mathrm{rad}}\right]^{T}\left[Y^{P}\right]\left[P_{t}^{p}\right],
$$

where $\left[P_{t}^{p}\right]$ is specified in the same form as $\left[P_{m, n}^{t}\right]$ of Eqs. (7) and (8), except that $p_{\text {total }}$ is replaced by $p_{\mathrm{pri}}^{0^{-}}$.

It should be noted that in practice, when designing an active noise control system, the panel velocity distribution under operating conditions can be evaluated in situ by modal decomposition. The resulting sound pressure distribution in the far field due to the primary source can then be estimated using Eq. (21), as the panel modal radiation transfer function matrix $\left[Z_{\mathrm{rad}}\right]$ is independent of the mechanical forcing functions acting upon the panel. Therefore, $p^{P}(\mathrm{r}, \omega)$ can be estimated directly from the panel velocity distribution, without knowing the details of the excitation forces.

\section{A. Vibration control sources}

Consider now the use of vibration secondary sources. Using Eqs. (4), (7), (8), (14), and (21), the total pressure level in the far field resulting from the control sources operating alone is

$$
\begin{aligned}
p^{S}(\mathbf{r}, \omega) & =\left[Z_{\mathrm{rad}}\right]^{T}\left[Y^{P}\right]\left[P_{t}^{s}\right] \\
& =\left[Z_{\mathrm{rad}}\right]^{T}\left[Y^{P}\right]\left[S^{\mathrm{con}}\right]\left[f^{\mathrm{con}}\right],
\end{aligned}
$$

where

$$
\left[f^{\mathrm{con}}\right]=\left(1 / A_{f}\right)\left[f_{1}^{\mathrm{con} \cdots} f_{M_{2}}^{\mathrm{con}}\right]^{T}
$$

and

$$
\left[S^{\mathrm{con}}\right]=\left(\begin{array}{ccc}
S_{1}\left(\sigma_{1}\right) & \cdots & S_{1}\left(\sigma_{M_{2}}\right) \\
\vdots & \ddots & \\
S_{M}\left(\sigma_{1}\right) & & S_{M}\left(\sigma_{M_{2}}\right)
\end{array}\right),
$$

where $S_{i}\left(\sigma_{j}\right)$ is the value of the mode shape function for the $i$ th mode at the location of the $j$ th control source.

Using Eqs. (30) and (31), Eq. (29) can be reexpressed as a quadratic function of the control force input:

$$
\begin{aligned}
|p(\mathbf{r}, \omega)|^{2}= & {\left[f^{\mathrm{con}}\right]^{H}[a]\left[f^{\mathrm{con}}\right]+\left[f^{\mathrm{con}}\right]^{H}[b] } \\
& +[b]^{H}\left[f^{\mathrm{con}}\right]+[c],
\end{aligned}
$$

where []$^{H}=[]^{* T}$ (the complex conjugate and transpose of a matrix),

$$
\begin{aligned}
& {[a]=\left[S^{\mathrm{con}}\right]^{H}[A]\left[S^{\mathrm{con}}\right],} \\
& {[A]=\left[Y^{P}\right]^{H}\left[Z_{\mathrm{rad}}\right]^{*}\left[Z_{\mathrm{rad}}\right]^{T}\left[Y^{P}\right],} \\
& {[b]=\left[S^{\mathrm{con}}\right]^{H}[A]\left[P_{t}^{p}\right],} \\
& {[c]=\left[P_{t}^{p}\right]^{H}[A]\left[P_{t}^{P}\right] .}
\end{aligned}
$$


corresponding to the optimum control force. This optimum value is

$$
\left[f_{\mathrm{opt}}^{\mathrm{con}}\right]=-[a]^{-1}[b] .
$$

The resulting minimum sound pressure is ${ }^{3}$

$$
|p(\mathbf{r}, \omega)|_{\min }^{2}=[c]-[b]^{H}[a]^{-1}[b] .
$$

It should be noted that Eqs. (39) and (40) are standard solutions to quadratic equations of the form of Eq. (34).

\section{B. Acoustic control sources}

A similar solution can be found for the use of acoustic control sources. The sound pressure at some point in the far field due to the acoustic control source(s) is given in Eq. (25). Using Eqs. (25) and (30), Eq. (29) can be rewritten as a quadratic function of the control source volume velocities

$$
\begin{aligned}
|p(\mathbf{r}, \omega)|^{2}= & {[u]^{H}\left[a_{A}\right][u]+[u]^{H}\left[b_{A}\right] } \\
& +\left[b_{A}\right]^{H}[u]+\left[c_{A}\right],
\end{aligned}
$$

where

$$
\begin{aligned}
& {\left[a_{A}\right]=\left[Z_{\text {mono }}\right]^{*}\left[Z_{\text {mono }}\right]^{T},} \\
& {\left[b_{A}\right]=\left[Z_{\text {mono }}\right]^{*}\left[Z_{\mathrm{rad}}\right]^{T}\left[Y^{P}\right]\left[P_{t}^{p}\right],} \\
& {\left[c_{A}\right]=\left[P_{t}^{p}\right]^{H}\left[Y^{P}\right]^{H}\left[Z_{\mathrm{rad}}\right]^{*}\left[Z_{\mathrm{rad}}\right]^{T}\left[Y^{P}\right]\left[P_{t}^{p}\right] .}
\end{aligned}
$$

[As stated previously, the modification of the panel velocity distribution by the inclusion of the acoustic control source(s) has been neglected as a second-order effect. If it does require consideration, an additional term can be included in $\left.\left[Z_{\text {mono }}\right].\right]$

Similar to the previous case of vibration control sources [see Eq. (39) ], the optimum control source volume velocity matrix is

$$
\left[u_{\mathrm{opt}}\right]=-\left[a_{A}\right]^{-1}\left[b_{A}\right]
$$

and the resulting minimum sound pressure at the error microphone is

$$
|p(\mathbf{r}, \omega)|_{\min }^{2}=\left[c_{A}\right]-\left[b_{A}\right]^{H}\left[a_{A}\right]^{-1}\left[b_{A}\right] .
$$

Once the optimum complex control forces or volume velocities for a particular control source/error microphone arrangement have been calculated, the resulting residual sound pressure level at any point can be found by using Eq. (29) with these control forces or volume velocities.

In a practical active noise control system, error sensors (usually microphones) sense the sound pressure level at a point or points and the inputs to the control actuators are adjusted by the controller to minimize this sensed "error signal." Normally, at least as many error sensors as control sources are required for there to be a unique combination of control source forces (or volume velocities) that will minimize the total squared sound pressure. For a noise source having some nonuniform directivity pattern, the placement of the "error microphone(s)" will have a significant effect upon the global attenuation achieved under optimum controlled conditions. Optimal controlled conditions refer to the control force amplitudes and phases necessary to minimize the sound pressure amplitudes of the error sensors. The total power optimization is usually limited at the computer simulation stage, because of the large number of sensors required. Therefore, when designing a system to actively control sound radiation from a structure, the contributions to the sound pressure level at the error sensing locations(s) from the various system components can be determined by the theory outlined in the previous sections. The sound pressure level at this point(s) can then be minimized, and the resulting residual radiation calculated. From this residual radiation, the total power radiated can be calculated and this power will be a function of the sensor location. The optimal location (s) of error sensor(s) for a particular control source arrangement can be determined by comparing the achieved total power reduction level (obtained by minimizing the farfield sound pressure) with that calculated by minimizing the total acoustic power. The calculation of the maximum achievable reduction of radiated sound power for a specified control source arrangement is discussed in the next section.

\section{MINIMIZATION OF TOTAL RADIATED SOUND POWER}

The maximum global noise attenuation possible for a given control source arrangement is found by minimizing the total system sound power output, which is given by ${ }^{12}$

$$
W_{\text {total }}=\int_{0}^{2 \pi} \int_{0}^{\pi / 2} \frac{|p(\mathbf{r}, \omega)|^{2}}{2 \rho_{0} c_{0}}|\mathbf{r}|^{2} \sin \theta d \theta d \phi_{r} .
$$

As in the previous section, the total acoustic power output can be expressed as a quadratic function of complex force inputs, for vibration control sources, or complex volume velocities, for acoustic control sources, if it is assumed that the acoustic control sources have infinite input impedances, and that the vibrational control sources have zero input impedances.

\section{A. Vibration control sources}

For the case of vibration control sources, the total acoustic power output can be expressed using the same series of operations as in the previous section:

$$
\begin{aligned}
W_{\text {total }}= & {\left[f^{\mathrm{con}}\right]^{H}[\bar{a}]\left[f^{\mathrm{con}}\right] } \\
& +\left[f^{\mathrm{con}}\right]^{H}[\bar{b}]+[\bar{b}]^{H}\left[f^{\mathrm{con}}\right]+[\bar{c}],
\end{aligned}
$$

where

$$
[\bar{a}]=\int_{0}^{2 \pi} \int_{0}^{\pi / 2} \frac{[a]}{2 \rho_{0} c_{0}} r^{2} \sin \theta d \theta d \phi_{r} .
$$

The same relationships exist between $[b],[c]$ and $[\bar{b}],[\bar{c}]$.

From Eq. (48), the optimum control force that minimizes the total sound power is

$$
\left[\bar{f}_{\mathrm{opt}}^{\mathrm{con}}\right]=-[\bar{a}]^{-1}[\bar{b}] \text {. }
$$

The resulting minimum achievable sound power output for the particular control source arrangement is

$$
W_{\text {total } \mid \text { min }}=[\bar{c}]-[\bar{b}]^{H}[\bar{a}]^{-1}[\bar{b}] .
$$

\section{B. Acoustic control sources}

Similarly, for the case of acoustic control sources, the total sound power can be expressed in terms of control source volume velocities: 


$$
\begin{aligned}
W_{\text {total }}= & {[u]^{H}\left[\bar{a}_{A}\right][u] } \\
& +[u]^{H}\left[\bar{b}_{A}\right]+\left[\bar{b}_{A}\right]^{H}[u]+\left[\bar{c}_{A}\right],
\end{aligned}
$$

where

$$
\left[\bar{a}_{A}\right]=\int_{0}^{2 \pi} \int_{0}^{\pi / 2} \frac{\left[a_{A}\right]}{2 \rho_{0} c_{0}} r^{2} \sin \theta d \theta d \phi_{r} .
$$

The same relationships exist between $\left[b_{A}\right],\left[c_{A}\right]$ and $\left[\bar{b}_{A}\right]$, $\left[\bar{c}_{A}\right]$.

Hence, the optimum volume velocities are

$$
\left[\bar{u}_{\text {opt }}\right]=-\left[\bar{a}_{A}\right]^{-1}\left[\bar{b}_{A}\right] \text {, }
$$

where $\left[\bar{u}_{\text {opt }}\right]$ is an $M_{2} \times 1$ matrix of the individual acoustic source volume velocities. This results in a minimum achievable sound power output of

$$
W_{\text {total } \min }=\left[\bar{c}_{A}\right]-\left[\bar{b}_{A}\right]^{H}\left[\bar{a}_{A}\right]^{-1}\left[\bar{b}_{A}\right],
$$

for a particular control source configuration.

\section{EXPERIMENTAL VERIFICATION}

\section{A. Experimental arrangement}

Experiments were conducted to verify the theory outlined in the preceding sections for the case of sound pressure minimization at a single point in the far field of the noise source using both acoustic and vibration control sources. The tests were undertaken in an anechoic chamber using a rectangular steel panel of $(x, y)$ dimensions $380 \mathrm{~mm} \times 300$ $\mathrm{mm} \times 2 \mathrm{~mm}$ thick mounted in a heavy steel frame. The steel

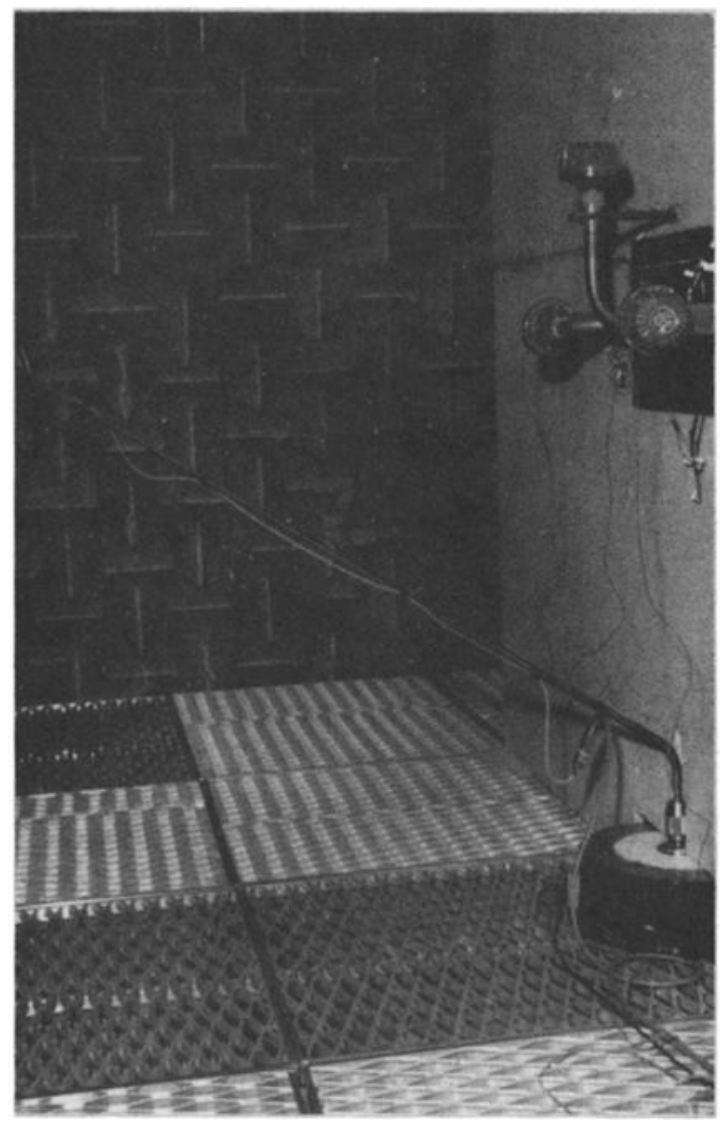

FIG. 2. Panel experimental arrangement. panel was placed in the center of a large, rigid wooden baffle of dimensions $4.8 \mathrm{~m} \times 2.4 \mathrm{~m} \times 19 \mathrm{~mm}$ thick, as shown in Fig. 2. Simply supported boundary conditions were implemented by using thin shim spring steel strips. One edge of each strip was attached to an edge of the panel by glue and small set screws while the other edge was bolted to the heavy steel frame. This approach gives a good approximation to the simply supported boundary condition as the shim is stiff for inplane motion but flexible for rotation. ${ }^{17}$

The panel was excited by a noncontacting electromagnetic exciter (primary source) located $3 \mathrm{~mm}$ from the plane of the panel, adjacent to the panel center at the rear. This driver consisted of a copper coil wound around an iron core (connected to the driving amplifier) surrounded by a permanent magnet. When a vibration secondary source was used, the control force was applied using an electrodynamic shaker connected to the panel just below the electromagnetic exciter with a 6-mm-diam by $250-\mathrm{mm}$-long aluminum rod, as shown in Fig. 3 (one attachment point was at $x=0 \mathrm{~mm}$, $y=-70 \mathrm{~mm}$ measured from the panel's center and the other was at $x=-150 \mathrm{~mm}, y=0 \mathrm{~mm}$ ). When acoustic control was used, each secondary source consisted of a horn driver attached to a 30-mm-diam tube with a $90^{\circ}$ bend and a flare to $50 \mathrm{~mm}$ at the open end, as shown in Fig. 2. This allowed the sound delivery to be positioned $20 \mathrm{~mm}$ away from the face of the panel with the minimum possible interference to the acoustic field.

The panel response was measured by using 17 accelerometers placed in two lines along the panel. Modal decomposition of the panel response was conducted by fitting the simply supported mode shape functions to the data, in a method similar to that described in Ref. 8. The radiated sound field was measured using a Bruel and Kjaer 1-in. microphone mounted on an arm attached to a turntable. This rotated through $180^{\circ}$ (from $-90^{\circ}-+90^{\circ}$ ), such that the microphone traversed a horizontal arc of $1.8-\mathrm{m}$ radius around the panel center, in a plane perpendicular to the plane of the panel, allowing the panel sound radiation directivity to be measured in one horizontal plane with a single microphone. The measurements were recorded as a polar plot using a Bruel and Kjaer level recorder.

The electromagnetic exciter was driven by a pure tone

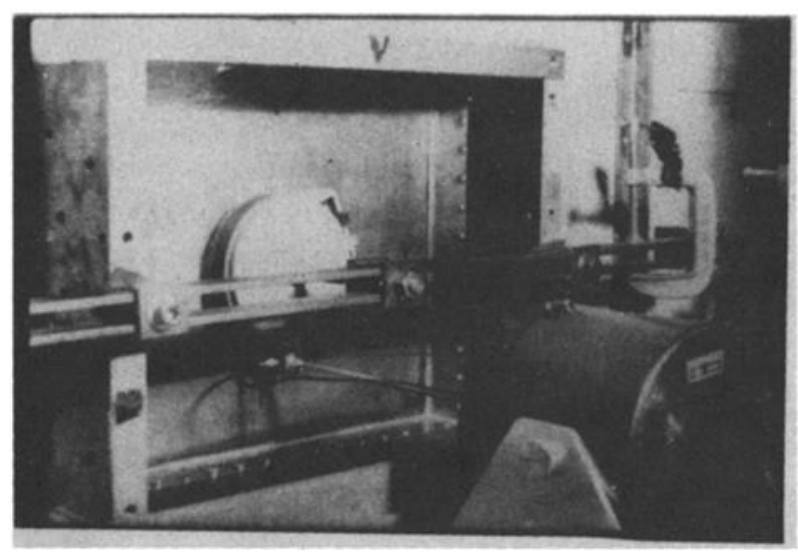

FIG. 3. Vibration control arrangement. 
reference signal. The same reference signal was used to drive the control source (vibration or acoustic) after being fed through a separate power amplifier and manually operated phase shifter. With both the primary exciter and control source(s) operating simultaneously, the amplitude and phase of the driving signal to the control exciter was adjusted to produce a minimum sound pressure level at a particular position of the traversing microphone. The residual panel radiation field and plate response were then measured.

The theoretical sound pressure distributions were calculated using the equations outlined in this paper. The electromagnetic actuator was modeled as a point force input at the panel center. This introduced some error into the results, as the forcing function contains a permanent distributed magnetic force component, as will be described later in this paper. For the particular control source arrangement, the sound pressure level at the error microphone position was minimized using the procedure outlined in Sec. IV. The residual sound field was then calculated at the radius of interest. The modal loss factors of the panel were determined by examination of the resonance peaks (as the resonances are well spaced and the panel is lightly damped) on a spectrum analyzer. For convenience, the average value of $\eta_{m, n}=0.0039$ so determined was used for all panel modes, as the values did not vary much between modes and because the final noise reduction predictions were not very sensitive to the loss factor.

For ease of comparison, the theoretical results were normalized to best fit the experimental data. This was done by matching the experimental and primary sound pressure fields by adding or subtracting the same constant value from each of the theoretically calculated data points. This same value was then added or subtracted from the theoretical controlled levels, allowing a direct assessment of the ability of the theoretical model to predict the residual controlled sound field. Further, for the theoretical results presented, the sound pressure reduction at the error microphone was limited to a value comparable to that achieved experimentally, and not reduced to the maximum level theoretically possible, to better simulate the practical system.

\section{B. Comparison of results: Vibration control sources}

The modal amplitudes resulting from the application of vibration control at $(x=0, y=-70) \mathrm{mm}$ with the sound pressure minimized at $(r, \phi, \theta)=\left(1.8,0^{\circ}, 0^{\circ}\right)$ are shown in Fig. 4. For this test, the excitation frequency was $338 \mathrm{~Hz}$, slightly below the $(2,2)$ and $(3,1)$ mode resonances, at approximately 351 and $359 \mathrm{~Hz}$, respectively. [Note that the $(1,1)$ mode is the fundamental mode.] In viewing the modal data it is clear that the principal mechanism here is one of modal amplitude control, where the amplitudes of the primary offending panel modes are significantly reduced. Corresponding experimental data are shown in Fig. 5 . It can be seen that experimentally the $(1,1)$ mode has not decreased as much as predicted, owing to the distributed nature of the primary force. This accounts for the 2-3-dB error in the radiation plot of Fig. 6, where the measured residual field is greater than that predicted theoretically. Despite this slight error, the agreement is good. The radiated sound fields, both

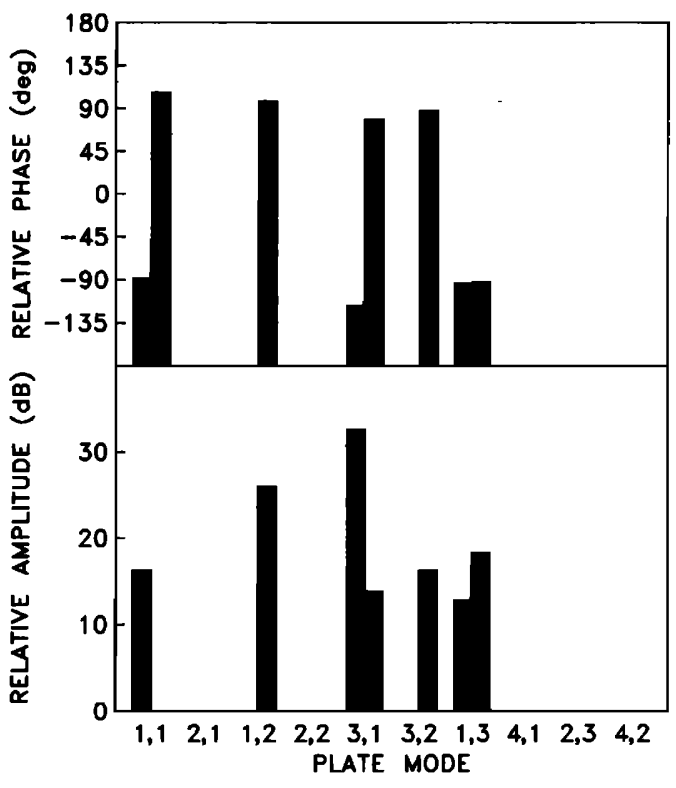

FIG. 4. Theoretical modal amplitudes and phases, vibration control at $(0,-70) \mathrm{mm}$, error sensor at $\left(1.8,0^{\circ}, 0^{\circ}\right)(\mathbb{G})=$ primary $(\square)=$ controlled. Excitation frequency $=338 \mathrm{~Hz}$.

controlled and uncontrolled and both predicted and measured, are shown in Fig. 6 for the cases corresponding to the modal distribution shown in Figs. 4 and 5, respectively.

In viewing Fig. 6 , it can be seen that the general agreement between theory and experiment is good. There are two main sources of experimental error, however. The first is the mass loading effect caused by the asymmetric placement of the accelerometers. This has the effect of slightly skewing the radiation plot. The second source of error is the distributed nature of the primary forcing function, which has the effect of slightly reducing the levels of attenuation achieved. This is because the action of the permanent magnetic force increases the input impedance for modes not having a nodal line across its center. The effect is worst for the $(1,1)$ mode, which has no nodal lines, and this makes it more resistant to change as a result of applying the vibration control force. Thus, if the amplitude of the mode should go up, it will go up

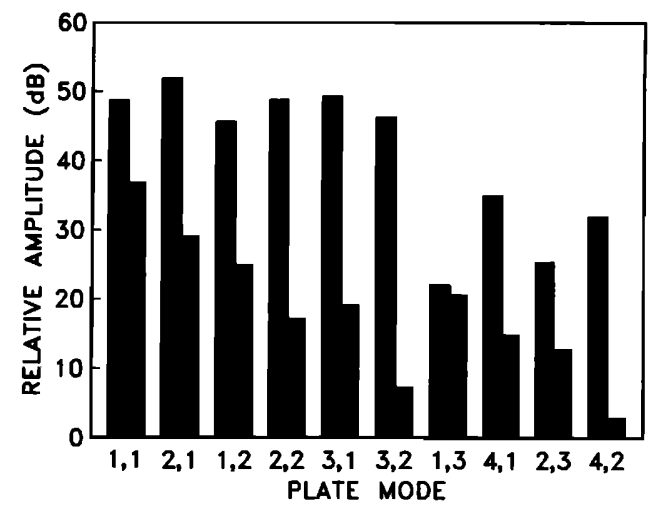

FIG. 5. Measured modal amplitudes, vibration control at $(0,-70) \mathrm{mm}$, error sensor at $\left(1.8,0^{\circ}, 0^{\circ}\right)\left(\square^{\prime}\right)=$ primary $(\square)=$ controlled. Excitation frequency $=338 \mathrm{~Hz}$. 


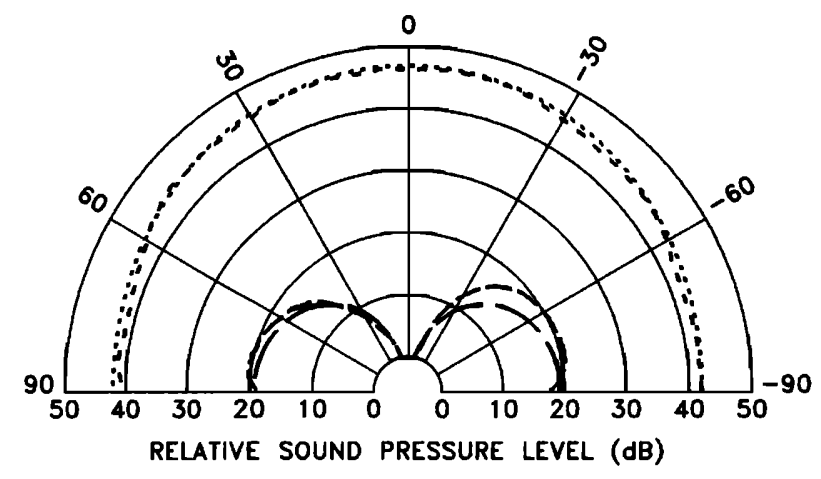

FIG. 6. Radiated sound pressure $1.8 \mathrm{~m}$ from the panel in a horizontal plane centered at the panel center; exciting frequency $=338 \mathrm{~Hz}$, vibration control at $(0,-70) \mathrm{mm}$, error sensor at $\left(1.8,0^{\circ}, 0^{\circ}\right), \cdots=$ theoretical primary, --$=$ measured primary, $-=$ theoretical controlled, $---=$ measured controlled. Total radiated power reduction $=10.5 \mathrm{~dB}$.

less than expected if the primary excitation were a point force; if it should go down, it will go down less than expected.

Note that the overall sound power level reduction corresponding to Fig. 6 is only $10.5 \mathrm{~dB}$, which is much smaller than might be expected by inspection of the sound pressure level reductions in the plane shown in the figure. This is because the below center location of the control source has excited the $(1,2)$ mode (see Fig. 4), which will contribute significantly to out of plane sound pressure levels.

Consider now the effect upon the modal amplitudes of applying vibration control at $(x=-150, y=0) \mathrm{mm}$, and minimizing the sound pressure at $\left(1.8,0^{\circ}, 0^{\circ}\right)$ (corresponding to the radiation plot of Fig. 9), shown in Fig. 7. Corresponding experimental data are shown in Fig. 8. In comparing Figs. 7 and 8, it can be seen that theoretically, the amplitude of the $(3,1)$ mode should be reduced, but that the amplitude of the $(1,1)$ mode should be increased. Here it is the mecha-

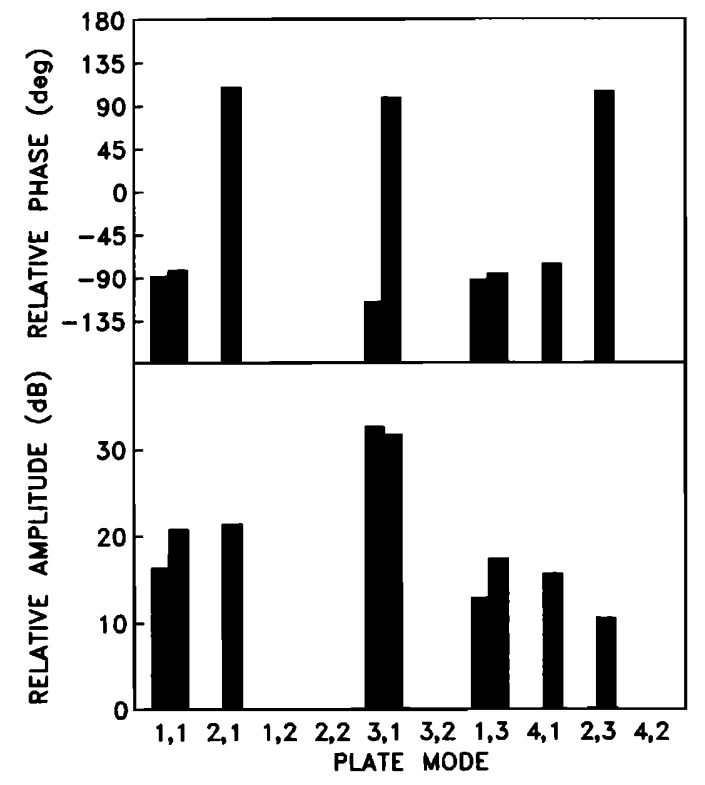

FIG. 7. Theoretical modal amplitudes and phases, vibration control at $(-150,0) \mathrm{mm}$, error sensor at $\left(1.8,0^{\circ}, 0^{\circ}\right)(\square)=$ primary $(\square)=$ controlled. Excitation frequency $=338 \mathrm{~Hz}$.

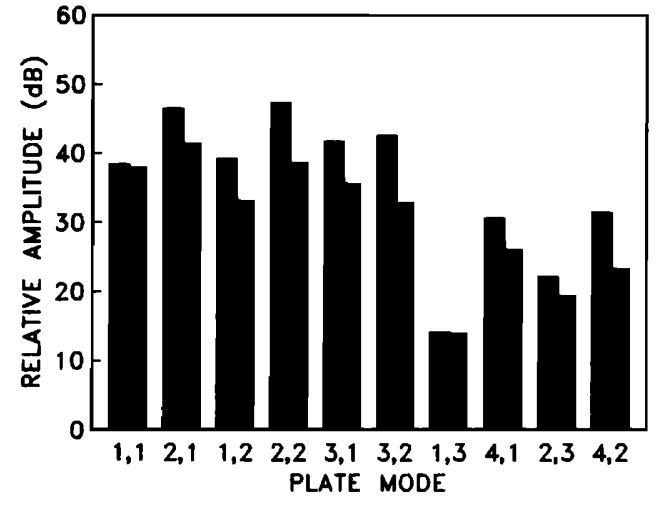

FIG. 8. Measured modal amplitudes, vibration control at $(-150,0) \mathrm{mm}$, error sensor at $\left(1.8,0^{\circ}, 0^{\circ}\right)(\square)=$ primary $(\square)=$ controlled. Excitation frequency $=338 \mathrm{~Hz}$.

nism of rearrangement of the amplitudes and phases of the panel modes that is providing sound control. Comparing this to the associated experimental result of Fig. 8, it can be seen that while the mechanism is still mainly one of modal amplitude and phase rearrangement, the predicted increase in the amplitude of the $(1,1)$ mode did not eventuate. This is again due to the influence of the distributed nature of the primary source, and accounts for the 2-3-dB error in the experimental radiation plots of Figs. 9 and 10, where the measured sound pressure level is less than that theoretically predicted. Despite this, the principal theoretical features can be seen clearly in the experimental data. In viewing Figs. 7 and 8, it can be seen that there is good agreement between measurement and prediction and the amplitudes of the panel modes have not been reduced markedly. This latter statement is especially true of the $(1,1)$ mode, which is one of the dominant radiators. To give an indication of the relative importance of the other modes, their radiation efficiencies are listed in Table I. It can be seen from Table I and Fig. 7 that the only important modes in terms of sound radiation are the $(1,1)$ and $(3,1)$ modes. However, one needs to take care in using this information. Although the overall sound pressure at a point can be observed by adding the contributions due to

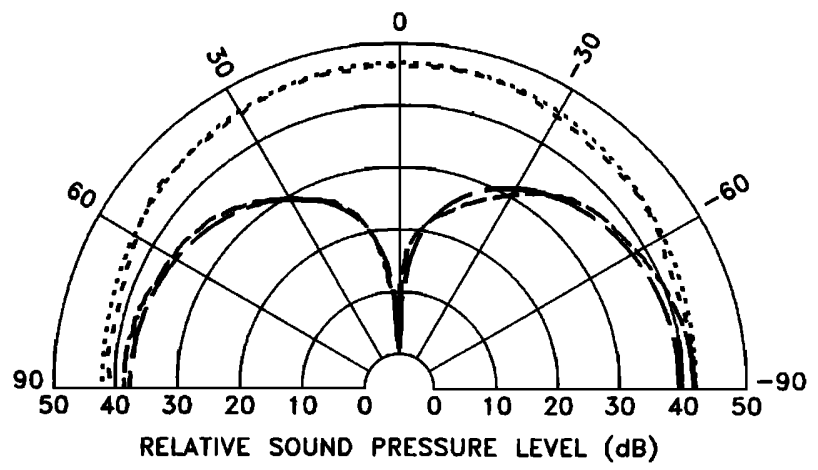

FIG. 9. Radiated sound pressure $1.8 \mathrm{~m}$ from the panel in a horizontal plane centered at the panel center; exciting frequency $=338 \mathrm{~Hz}$, vibration control at $(-150,0) \mathrm{mm}$, error sensor at $\left(1.8,0^{\circ}, 0^{\circ}\right), \cdots=$ theoretical primary, $\cdots$ $=$ measured primary, $-=$ theoretical controlled, $---=$ measured controlled. Total radiated power reduction $=12.8 \mathrm{~dB}$. 


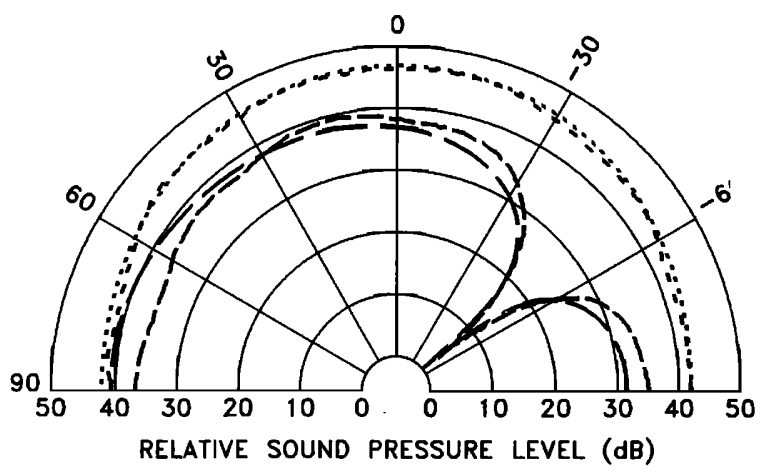

FIG. 10. Radiated sound pressure $1.8 \mathrm{~m}$ from the panel in a horizontal plane centered at the panel center; exciting frequency $=338 \mathrm{~Hz}$, vibration control at $(0,-70) \mathrm{mm}$, error sensor at $\left(1.8,0^{\circ}, 50^{\circ}\right), \cdots=$ theoretical primary, $-\cdots$ $=$ measured primary, $-=$ theoretical controlled, $---=$ measured controlled. Total radiated power reduction $=8.5 \mathrm{~dB}$.

each mode, the sound power cannot be obtained by adding together the individual power attributable to each mode, as pressure squared quantities that ignore relative phases cannot be combined in this way. To achieve the levels of radiated sound pressure (hence power) attenuation seen in Fig. 9, rearrangement of the modal amplitudes and phases must be the mechanism at work. The difference in primary field modal distributions for the two different control source locations shown in Figs. 6 and 9 is due to loading of the panel by the control shaker that was attached, but not operational.

Plots of the theoretical and measured primary radiated and residual controlled sound fields corresponding to the modal plots of Figs. 7 and 8 are given in Fig. 9. The effect of moving the error sensor to $\left(1.8,0^{\circ}, 50^{\circ}\right)$ is shown in Fig. 10. In viewing these, it is again clear that the agreement between theory and experiment is good. However, a notable feature of both plots is that there is a 2-3-dB difference between the theoretical and measured residual sound fields, with the amplitudes of the measured residual sound fields being less than those predicted theoretically. This error is due predominantly to the distributed nature of the primary forcing function, as was discussed previously.

It is clear from viewing Figs. 6, 9, and 10 that significant levels of reduction in the total radiated sound power have been achieved, with the vibration control source at two different locations. As mentioned earlier, there are two possible mechanisms of control when vibration sources are used;

TABLE I. Modal radiation efficiencies at $338 \mathrm{~Hz}$.

\begin{tabular}{ll}
\hline \hline Mode & \multicolumn{1}{c}{$\boldsymbol{\sigma}$} \\
\hline 1,1 & 0.397 \\
2,1 & 0.046 \\
1,2 & 0.029 \\
2,2 & 0.0020 \\
3,1 & 0.032 \\
3,2 & 0.0027 \\
1,3 & 0.036 \\
4,1 & 0.010 \\
2,3 & 0.0045 \\
4,2 & 0.0005 \\
\hline \hline
\end{tabular}

modal control, where the amplitudes of the dominant radiating panel modes are reduced, and modal rearrangement, where the relative amplitudes and phases of the dominant panel modes are altered, so as to reduce the overall radiation efficiency of the panel. These two mechanisms can coexist, and do so in varying degrees, for any given location of the vibration control application.

In contrast to Fig. 6 , the sound power reduction of 12.8 $\mathrm{dB}$ corresponding to Fig. 9 is in general agreement with the sound pressure level reductions shown. This is because in this case, the $(1,2)$ mode has not been significantly excited, as the control source is at $y=0$ (a nodal line for this mode). Similar comments can be made about the sound field shown in Fig. 10 and the corresponding sound power reduction of $8.5 \mathrm{~dB}$.

To better understand how the modal amplitude and phase rearrangement mechanism provides global farfield sound attenuation, it is useful to consider the theoretical primary and controlled surface velocity amplitudes and phases

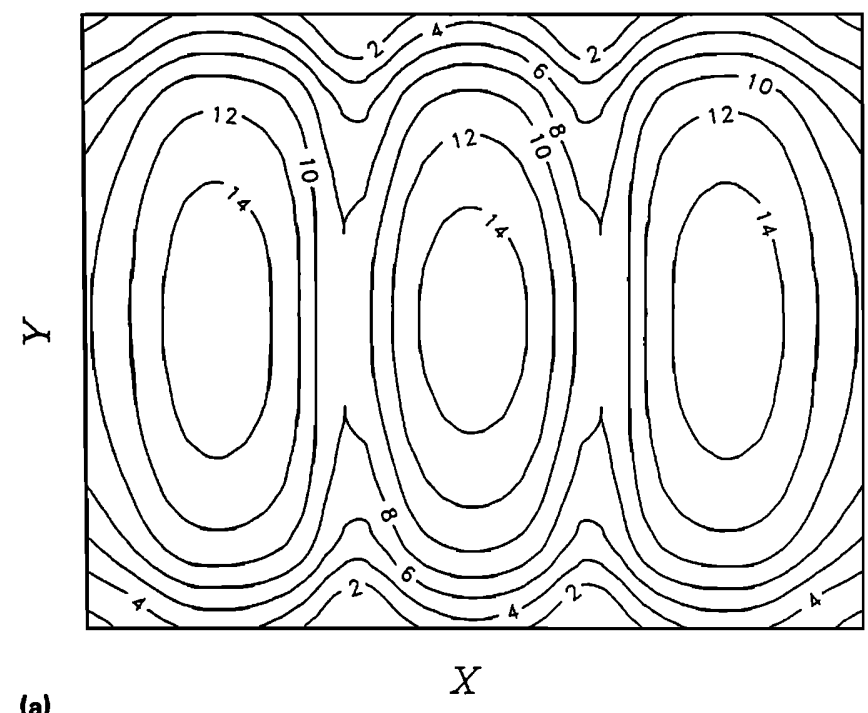

(a)

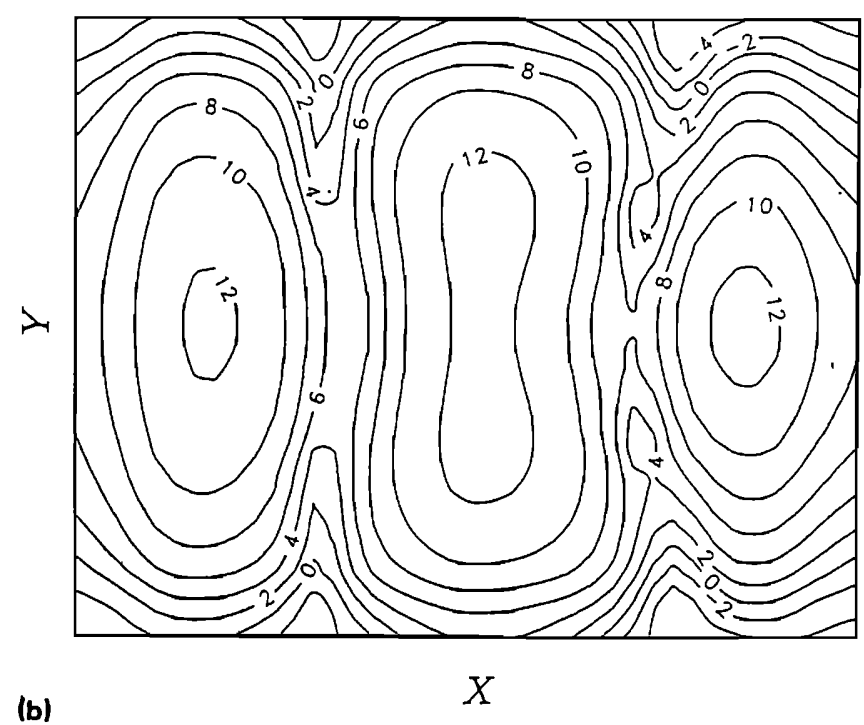

FIG. 11. Theoretical mean square velocity levels $(\mathrm{dB}), f=338 \mathrm{~Hz}$. (a) Primary, (b) controlled. 
associated with the modal plot of Fig. 7. These amplitudes and phases are shown in Figs 11 and 12. Comparing the primary and controlled velocity distribution plots of Fig. 12(a) and (b), it can be seen that the surface velocity does decrease under the action of active vibration control, but only by approximately $2 \mathrm{~dB}$. This is not, however, enough to account for the large reduction in the radiated sound pressure.

When this result is viewed in light of the change in the phasing of the surface velocity, found by comparing the primary phasing of Fig. 12(a) with the controlled phasing of Fig. 12(b), the total effect can be deduced. Under the action of vibration control the "high velocity" center region of the panel, which is approximately $180^{\circ}$ out of phase with each side, has increased in size relative to the two edge regions. Thus the center region has become more of an acoustic sink, reducing the overall radiation efficiency of the panel.

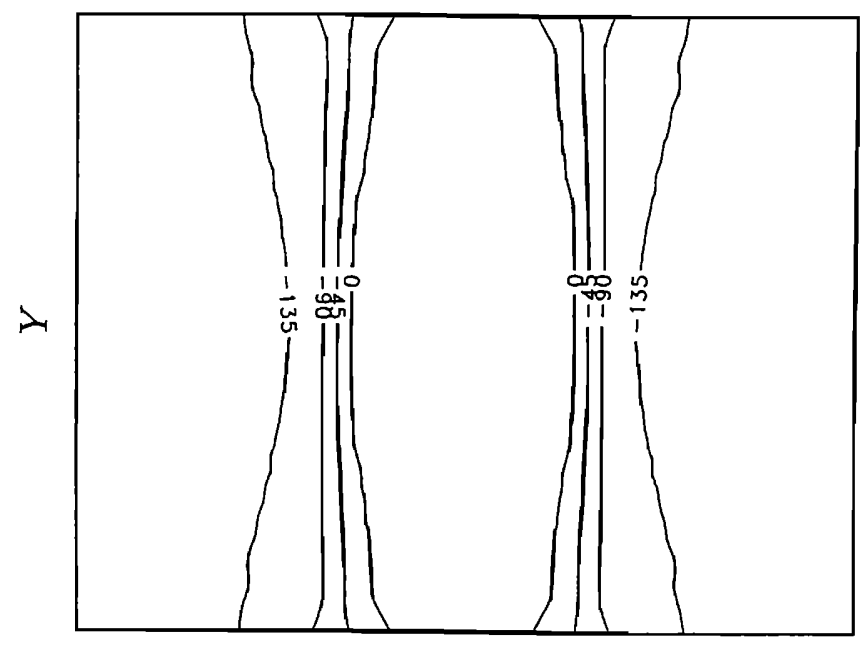

(a) $X$

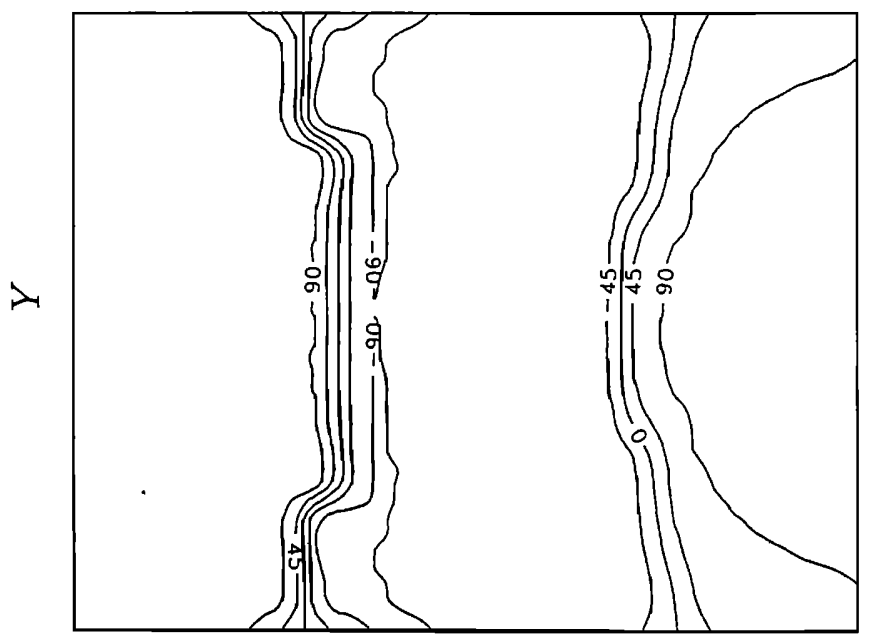

(b)

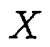

FIG. 12. Theoretical primary phasing (deg), $f=338 \mathrm{~Hz}$. (a) Primary, (b) controlled.

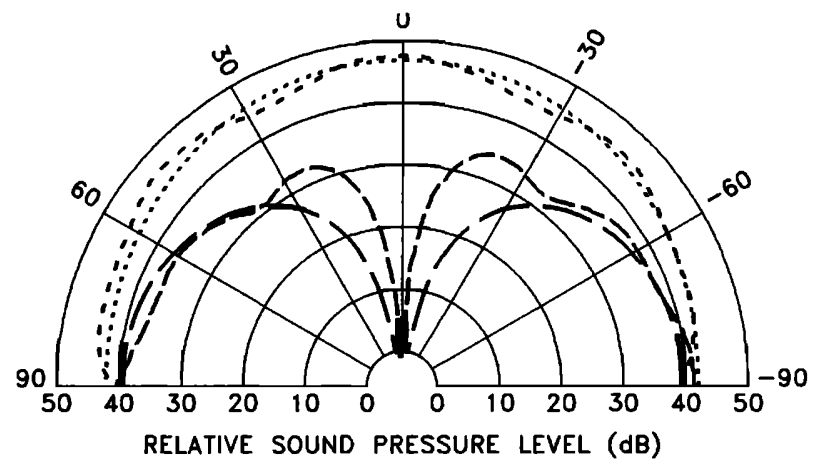

FIG. 13. Radiated sound pressure $1.8 \mathrm{~m}$ from the panel in a horizontal plane centered at the panel center; exciting frequency $=338 \mathrm{~Hz}$, acoustic control at $(0,0) \mathrm{mm}$, error sensor at $\left(1.8,0^{\circ}, 0^{\circ}\right), \cdots=$ theoretical primary, $\cdots$ $=$ measured primary, $-=$ theoretical controlled, $---=$ measured controlled. Total radiated power reduction $=10.7 \mathrm{~dB}$.

\section{Comparison of results: Acoustic control sources}

The use of acoustic control sources was examined next. A single horn driver (the center one in Fig. 2), discharging at the panel center, was used to control the acoustic pressure radiated from the 2-mm-thick panel vibrating at $338 \mathrm{~Hz}$. The comparison between theory and experiment for the cases of minimizing the sound pressure at an angular location of $90^{\circ}$ and $40^{\circ}$ is shown in Figs. 13 and 14, respectively. In viewing these it can be seen that the general agreement between theory and experiment is good, although diffraction around the horn driver has slightly altered the acoustic field, introducing an interference pattern into the result. Using three rather than one horn drivers resulted in a more pronounced interference pattern due to diffraction around the additional drivers and slightly better noise reduction results were obtained, as might be expected.

The total reductions in sound power levels of 10.7 and $15.0 \mathrm{~dB}$ are in general agreement with the average in-plane sound pressure levels shown in Figs. 13 and 14, respectively, suggesting that the acoustic control source does not excite any additional out of plane plate modes.

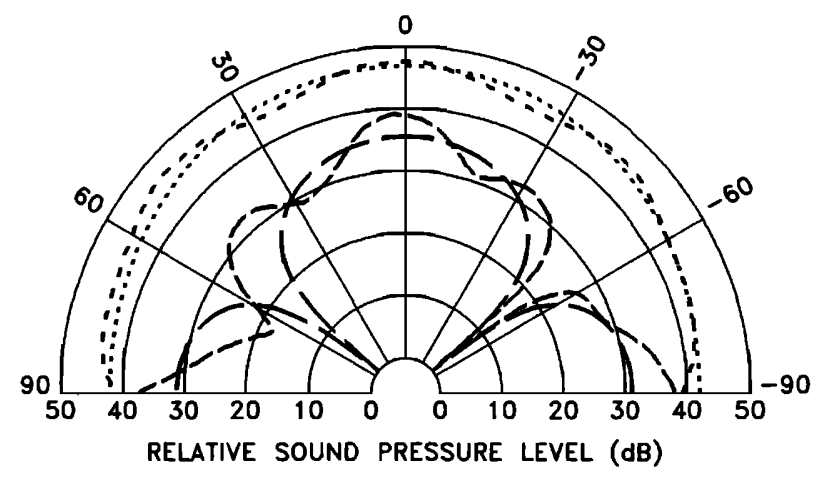

FIG. 14. Radiated sound pressure $1.8 \mathrm{~m}$ from the panel in a horizontal plane centered at the panel center; exciting frequency $=338 \mathrm{~Hz}$, acoustic control at $(0,-70) \mathrm{mm}$, error sensor at $\left(1.8,0^{\circ}, 50^{\circ}\right), \cdots=$ theoretical primary, --$=$ measured primary, $-=$ theoretical controlled, $---=$ measured controlled. Total radiated power reduction $=15.0 \mathrm{~dB}$. 


\section{CONCLUSIONS}

A theoretical model for predicting the reduction in sound power radiated by a harmonically vibrating simply supported panel, by application of either vibration or acoustic control sources has been verified experimentally. The model shows that active noise control can produce global sound attenuation by decreasing the acoustic power flow from the offending primary noise source, and when the primary source approximates a baffled, simply supported panel, either acoustic or vibration control sources are effective. Acoustic control sources affect the in-phase component of the surface pressure, thereby effectively modifying the total radiation impedance seen by the panel. Vibration control sources modify the panel velocity distribution by either increasing the input impedance of the offending modes, hence decreasing their amplitude, and/or changing the temporal phases of the panel modes, hence effectively changing the total radiated power of the panel.

The results presented here show that, for a given panel and primary exciting force, the employment of these two mechanisms can be combined in varying degrees at any given vibration control application point. Also, a single vibration control source can utilize effectively either or both of these mechanisms in some instances.

It is interesting to consider that a single vibration control source can reduce the amplitudes of a number of structural modes simultaneously, without causing an increase in the amplitudes of other structural modes. This can be explained by considering that for a lightly damped, mechanically excited panel there will be regions where the majority of the primary radiating structural modes will be in phase. In these regions, a single vibration source can effectively control all of the in-phase modes simultaneously to some degree, dependent upon the relative vibration amplitudes of the modes. Conversely, it is the use of a control source at locations on the panel where the primary structural modes are out of phase, which results in the structural modal rearrangement control mechanism being important in providing sound attenuation.

\section{ACKNOWLEDGMENTS}

Support for this work from the Sir Ross and Sir Keith Smith Fund, NASA Langley Research Center, Grant No.
NAG1-390, and the University of Adelaide is gratefully acknowledged.

${ }^{1}$ R. R. Leitch and M. O. Tokhi, "Active noise control systems," IEE Proc. Pt. A 134, 525-546 (1987).

${ }^{2}$ S. D. Snyder and C. H. Hansen, "Active noise control in ducts: Some physical insights," J. Acoust. Soc. Am. 86, 184-194 (1989).

${ }^{3}$ P. A. Nelson, A. R. D. Curtis, S. J. Elliott, and A. J. Bullmore, "The minimum power output of free field point sources and the active control of sound," J. Sound Vib. 116, 397-414 (1987).

${ }^{4} \mathrm{~N}$. Hesselmann, "Investigation of noise reduction on a $100 \mathrm{kVA}$ transformer tank by means of active methods," Appl. Acoust. 11, 27-34 (1978).

${ }^{5}$ C. R. Fuller and J. D. Jones, "Experiments on reduction of propeller induced interior noise by active control of cylinder vibration," J. Sound Vib. 112, 389-395 (1987).

${ }^{6} \mathrm{P}$. Eatwell, "Active control of automobile cabin noise," Proceedings of Inter-Noise 89, Newport Beach.

${ }^{7}$ T. Berge, O. Kr. O. Petterson, and S. Sorzdal, "Active cancellation of transformer noise: Field measurements," Appl. Acoust. 23, 309-320 (1988).

${ }^{8}$ E. K. Dimitriadis and C. R. Fuller, "Investigation on active control of sound transmission through elastic plates using Piezoelectric actuators," AIAA Conference Paper No. AIAA-89-1062, 1989.

${ }^{9}$ C. R. Fuller, C. H. Hansen, and S. D. Snyder, "Active control of structurally radiated noise using piezoelectric actuators," Inter-Noise '89, Long Beach, CA, 1989.

${ }^{10}$ C. H. Hansen, S. D. Snyder, and C. R. Fuller, "Reduction of Noise Radiated by a vibrating rectangular panel by active sound sources and active vibration sources: A comparison," Proceedings of Noise and Vibration, 1989, Singapore.

${ }^{11}$ J. Pan, C. H. Hansen, and D. A. Bies, "Active control of noise transmission through a panel into a cavity: I. Analytical study," J. Acoust. Soc. Am. 87, 2098-2108 (1990).

${ }^{12}$ C. Deffayet and P. A. Nelson, "Active control of low-frequency harmonic sound radiation by a finite panel," J. Acoust. Soc. Am. 84, 2192-2199 (1988).

${ }^{13} \mathrm{C}$. R. Fuller, "Analysis of active control of sound radiation from elastic plates by force inputs," Inter-Noise '88, Avignon, France, August, 1988.

${ }^{14}$ P. M. Morse and K. U. Ingard, Theoretical Acoustics (McGraw-Hill, New York, 1968).

${ }^{15}$ C. E. Wallace, "Radiation resistance of a rectangular panel," J. Acoust. Soc. Am. 51, 946-952 (1972).

${ }^{16}$ P. A. Nelson and S. J. Elliott, "Active minimisation of acoustic fields," J. Theor. Appl. Mech. Special issue, supplement to 6 (1987).

${ }^{17} \mathrm{~J}$. Ochs and J. Snowdon, "Transmissibility across simply supported thin plates, 1. Rectangular and square plates with and without damping layers," J. Acoust. Soc. Am. 58, 832-840 (1975). 